\title{
Asymptotic Behavior for Second Order Lattice Dynamical Systems
}

\author{
SHENGFAN ZHOU \\ Department of Mathematics, Sichuan University, Chengdu 610064, People's Republic of China
}

(Received 10 April 2000)

We consider the existence of the global attractor for a second order lattice dynamical systems.

Keywords: Global attractor; Lattice dynamical system; Equivalent norm

\section{INTRODUCTION}

Recently, there are more and more authors to study the various properties of solutions for lattice dynamical systems, mainly are coupled map lattices and lattice ordinary differential equations, see $[1-5]$ and the references therein. Lattice systems can be found in many fields of applications, for example, in chemical reaction theory, image processing and pattern recognition. Lattice systems have their own forms, in some cases, they arise in the spatially discretizations of partial differential equations.

In this paper, we shall consider the asymptotic behavior of solutions for the following second order lattice dynamical system:

$$
\underset{\ddot{u}_{i}+\alpha \dot{u}_{i}-\left(u_{i-1}-2 u_{i}+u_{i+1}\right)+\lambda u_{i}+\underset{i \in Z}{f}\left(u_{i}\right)=g_{i},}{i(1)}
$$

where $\alpha$ and $\lambda$ is a positive constants, $g_{i}$ is given, $f(s)=\sum_{j=0}^{m} a_{j} s^{2 j+1}$ with $a_{j}>0, j=0,1, \ldots, m$, is a polynomial. By introducing a new weight inner product and norm in the space $\ell^{2}=\left\{u=\left(u_{i}\right)_{i \in Z}\right.$ $\left.\mid u_{i} \in R, \sum_{i \in Z} u_{i}^{2}<\infty\right\}$, we prove the existence of a global attractor of system (1). The idea of using such a technique is due to Zhou [6] and Bates $^{1}$, the later considered the existence of a global attractor for a first order lattice dynamical system.

Equation (1) can be regarded as a discrete analogue of the following continuous damped semi-linear wave equation:

$$
u_{t t}+\alpha u_{t}-u_{x x}+\lambda u+f(u)=g .
$$

The global attractor and its dimension to Eq. (2) in bounded domain and unbounded domain have been studied in Hilbert spaces by

\footnotetext{
${ }^{1}$ Peter W. Bates, Kening Lu, Bixiang Wang, Attractors for lattice dynamical systems, Preprint, 1999.
} 
many people, see [6-12] and the references therein.

This paper is organized as follows. In the second section, we present the existence and uniqueness of solutions for system (1). In Section 3, we prove the uniformly boundedness of solutions. In Section 3, we prove the existence of the global attractor.

\section{EXISTENCE AND BOUNDEDNESS OF SOLUTIONS}

In this section, we consider the existence and uniqueness of solutions for system (1) with initial conditions:

$$
\left\{\begin{array}{l}
\ddot{u}_{i}+\alpha \dot{u}_{i}-\left(u_{i-1}-2 u_{i}+u_{i+1}\right)+\lambda u_{i}+f\left(u_{i}\right)=g_{i} \\
u_{i}(0)=u_{i, 0}, \quad \dot{u}_{i}(0)=u_{1 i, 0}, \quad i \in Z
\end{array}\right.
$$

where $\alpha, \lambda>0, g=\left(g_{i}\right)_{i \in Z}$ and $f(s)=\sum_{j=0}^{m} a_{j} s^{2 j+1}$ with $a_{j}>0, j=0,1, \ldots, m$. For any $u=\left(u_{i}\right)_{i \in Z} \in \ell^{2}$, define

$$
\begin{aligned}
& (B u)_{i}=u_{i+1}-u_{i}, \quad(\bar{B} u)_{i}=u_{i-1}-u_{i} \\
& (A u)_{i}=-\left(u_{i-1}-2 u_{i}+u_{i+1}\right), \quad \forall i \in Z .
\end{aligned}
$$

Then $B, \bar{B}, A$ are linear operators from $\ell^{2}$ to $\ell^{2}$ and satisfy $A=\bar{B} B=B \bar{B}$.

For any two elements $u=\left(u_{i}\right)_{i \in Z}, v=\left(v_{i}\right)_{i \in Z}$ $\in \ell^{2}$, define two bilinear forms as

$$
\left\{\begin{array}{l}
(u, v)=\sum_{i \in Z} u_{i} v_{i},\|u\|^{2}=(u, u)=\sum_{i \in Z}\left|u_{i}\right|^{2} ; \\
(u, v)_{\lambda}=(B u, B v)+\lambda(u, v), \\
\|u\|_{\lambda}^{2}=(u, u)_{\lambda}=\|B u\|^{2}+\lambda\|u\|^{2}=\sum_{i \in Z}\left(\left|u_{i+1}-u_{i}\right|^{2}+\lambda\left|u_{i}\right|^{2}\right) .
\end{array}\right.
$$

Obviously, the bilinear forms $(\cdot, \cdot)$ and $(\cdot, \cdot)_{\lambda}$ in (4) are both the inner products, moreover, the norms $\|\cdot\|$ and $\|\cdot\|_{\lambda}$ are equivalent each other because

$$
\begin{aligned}
\lambda\|u\|^{2} & \leq\|u\|_{\lambda}^{2}=\sum_{i \in Z}\left(\left|u_{i+1}-u_{i}\right|^{2}+\lambda\left|u_{i}\right|^{2}\right) \\
& \leq(4+\lambda)\|u\|^{2} .
\end{aligned}
$$

Denote by $\ell^{2}, \ell_{\lambda}^{2}$ the spaces with the inner products and norms in (4), respectively $\ell^{2}=\left(\ell^{2},(\cdot, \cdot),\|\cdot\|\right)$, $\ell_{\lambda}^{2}=\left(\ell^{2},(\cdot, \cdot)_{\lambda},\|\cdot\|_{\lambda}\right)$, then $\ell^{2}$ and $\ell_{\lambda}^{2}$ are Hilbert spaces. Let $E=\ell_{\lambda}^{2} \times \ell^{2}$, endowed with the inner product and norm as: for $\varphi_{j}=\left(u^{(j)}, v^{(j)}\right)=\left(\left(u_{i}^{(j)}\right)\right.$, $\left.\left(u_{i}^{(j)}\right)\right)_{i \in Z} \in E, j=1,2$,

$$
\begin{aligned}
&\left(\varphi_{1}, \varphi_{2}\right)_{E}=\left(u^{(1)}, u^{(2)}\right)_{\lambda}+\left(v^{(1)}, v^{(2)}\right) \\
&= \sum_{i}\left[\left(B u^{(1)}\right)_{i}\left(B u^{(2)}\right)_{i}\right. \\
&\left.\quad+\lambda u_{i}^{(1)} u_{i}^{(2)}+v_{i}^{(1)} v_{i}^{(2)}\right] \\
&\|\varphi\|_{E}^{2}=(\varphi, \varphi)_{E}, \quad \forall \varphi \in \ell_{\lambda}^{2} \times \ell^{2}
\end{aligned}
$$

It is convenient to reduce system (3) as an ordinary differential equation of first order in time on $E$. With above notations, problem (3) can be written as

$$
\left\{\begin{array}{l}
\ddot{u}+\alpha \dot{u}+A u+\lambda u+f(u)=g, \quad t>0, \\
u(0)=\left(u_{i, 0}\right)_{i \in Z}=u_{0}, \quad \dot{u}(0)=\left(u_{1 i, 0}\right)_{i \in Z}=u_{10},
\end{array}\right.
$$

where $u=\left(u_{i}\right)_{i \in Z}, f(u)=\left(f\left(u_{i}\right)\right)_{i \in Z}, \quad g=\left(g_{i}\right)_{i \in Z}$. Let $v=\dot{u}+\varepsilon u$, where $\varepsilon$ is chosen as

$$
\varepsilon=\frac{\alpha \lambda}{\alpha^{2}+4 \lambda}>0
$$

then system (6) is equivalent to the following initial value problem in Hilbert space $E$

$$
\begin{aligned}
\dot{\varphi}+C \varphi & =F(\varphi), \\
\varphi(0) & =\left(u_{0}, v_{0}\right)^{T}=\left(u_{0}, u_{10}+\varepsilon u_{0}\right)^{T},
\end{aligned}
$$

where $\varphi=(u, v)^{T}, \quad v=\dot{u}+\varepsilon u, \quad F(\varphi)=(0,-$ $f(u)+g)^{T}$,

$$
C=\left(\begin{array}{cc}
\varepsilon I & -I \\
A+\lambda I+\varepsilon(\varepsilon-\alpha) I & (\alpha-\varepsilon) I
\end{array}\right) .
$$

For any $u=\left(u_{i}\right)_{i \in z} \in \ell^{2},\left|u_{i}\right| \leq\|u\|$,

$$
\|f(u)\|=\left(\sum_{i \in Z}\left|f\left(u_{i}\right)\right|^{2}\right)^{1 / 2} \leq\|u\| \sum_{j=0}^{m} a_{i}\|u\|^{2 j},
$$


thus, $f$ maps $\ell^{2}$ into $\ell^{2}$, i.e., $F$ maps $E$ into itself. Let $B$ be a bounded set in $E, \varphi_{j}=\left(u^{(j)}, v^{(j)}\right)=$ $\left(\left(u_{i}^{(j)}\right),\left(v_{i}^{(j)}\right)\right)_{i \in Z} \in B, j=1,2$, similar to $(10)$, there exists $L\left(a_{i}, B\right)$ such that

$$
\left\|F\left(\varphi_{1}\right)-F\left(\varphi_{2}\right)\right\|_{E} \leq L\left(a_{i}, B\right)\left\|\varphi_{1}-\varphi_{2}\right\|_{E}
$$

thus, $F(\varphi)$ is locally Lipschitz from $E$ to $E$. It is easy to see that the solutions of problem (3) is backward unique in time because if $t$ and $\alpha$ are replaced by $-t$ and $-\alpha$, the Eq. (3) is not changed. By the standard theory of ordinary differential equations, we obtain the existence and uniqueness of local solution $\varphi$ for problem (8).

Lemma 1 If $g=\left(g_{i}\right)_{i \in Z} \in \ell^{2}$, then for any initial data $\varphi(0)=\left(u_{0}, v_{0}\right)^{T} \in E$, there exists an unique local solution $\varphi(t)=(u(t), v(t))^{T}$ of (8) such that $\varphi \in C^{1}$ $\left(\left(-T_{0}, T_{0}\right), E\right)$ for some $T_{0}>0$. If $T_{0}<+\infty$, then $\lim _{t \rightarrow T_{0}}\|\varphi(t)\|_{E}=+\infty$.

From Lemma 3 below, it is obtained that the local solution $\varphi(t)$ of (8) exists globally, that is, $\varphi \in C^{1}(R, E)$, which implies that maps

$$
\begin{aligned}
S(t): \varphi(0) & =\left(u_{0}, v_{0}\right) \in E \rightarrow \varphi(t)=S(t) \varphi(0) \\
& =(u(t), v(t)) \in E, \quad t \geq 0
\end{aligned}
$$

generates a continuous semigroup $\{S(t)\}_{t \geq 0}$ on $E$, where $v(t)=\dot{u}(t)+\varepsilon u(t)$.

\section{BOUNDEDNESS OF SOLUTIONS}

Lemma 2 For any $\varphi=(u, v)^{T} \in E$,

$$
(C \varphi, \varphi)_{E} \geq \sigma\|\varphi\|_{E}^{2}+\frac{\alpha}{2}\|v\|^{2}
$$

where

$$
\sigma=\frac{\alpha \lambda}{\sqrt{\alpha^{2}+4 \lambda}\left(\alpha+\sqrt{\alpha^{2}+4 \lambda}\right)} .
$$

Proof It is easy to check that

$$
\begin{aligned}
& (B u, v)=(u, \bar{B} v) \quad \text { and } \\
& (A u, v)=(B u, B v), \quad \forall u, v \in \ell^{2} .
\end{aligned}
$$

and

$$
\begin{aligned}
& (C \varphi, \varphi)_{E}-\sigma\|\varphi\|_{E}^{2}-\frac{\alpha}{2}\|v\|^{2} \\
& \geq(\varepsilon-\sigma)\left[\|B u\|^{2}+\lambda\|u\|^{2}\right] \\
& \quad+\left(\frac{\alpha}{2}-\varepsilon-\sigma\right)\|v\|^{2} \\
& \quad-\frac{\alpha \varepsilon}{\sqrt{\lambda}}\left[\|B u\|^{2}+\lambda\|u\|^{2}\right]^{1 / 2}\|v\|,
\end{aligned}
$$

But

$$
4(\varepsilon-\sigma)\left(\frac{\alpha}{2}-\varepsilon-\sigma\right)=\frac{\alpha^{2} \varepsilon^{2}}{\lambda}
$$

Thus, the proof is completed.

We consider the boundedness of solutions $\varphi(t)$ of (8). Assume that $g \in \ell^{2}$. Let $\varphi(t)=$ $(u(t), v(t))^{T} \in E$ be a solution of (8), where $v(t)=$ $\dot{u}(t)+\varepsilon u(t)$.

Taking the inner product $(\cdot, \cdot)_{E}$ of (8) with $\varphi(t)$, we have

$\frac{1}{2} \frac{d}{d t}\|\varphi\|_{E}^{2}+(C \varphi, \varphi)_{E}+(f(u), \dot{u})+\varepsilon(f(u), u)=(g, v)$.

By (12),

$$
(C \varphi, \varphi)_{E} \geq \sigma\|\varphi\|_{E}^{2}+\frac{\alpha}{2}\|v\|^{2}
$$

Write $G(s)=\int_{0}^{s} f(\tau) d \tau=\sum_{j=0}^{m}\left(a_{j} /(2 j+2)\right) s^{2 j+2}$, then

$$
(f(u), \dot{u})=\sum_{i \in Z} f\left(u_{i}\right) \dot{u}_{i}=\frac{d}{d t}\left(\sum_{i \in Z} G\left(u_{i}\right)\right)
$$

$$
(f(u), u)=\sum_{i \in Z} f\left(u_{i}\right) u_{i} \geq \sum_{i \in Z} G\left(u_{i}\right),
$$


and

$$
(g, v) \leq \frac{1}{2 \alpha}\|g\|^{2}+\frac{\alpha}{2}\|v\|^{2} .
$$

By putting (15) - (18) into (14), we find

$$
\begin{aligned}
& \frac{d}{d t}\left[\|\varphi\|_{E}^{2}+2 \sum_{i \in Z} G\left(u_{i}\right)\right] \\
& \quad+\sigma\left[\|\varphi\|_{E}^{2}+2 \sum_{i \in Z} G\left(u_{i}\right)\right] \leq \frac{1}{\alpha}\|g\|^{2} .
\end{aligned}
$$

By Gronwall's inequality,

$$
\begin{aligned}
\|\varphi\|_{E}^{2} & +2 \sum_{i \in Z} G\left(u_{i}\right) \\
\leq & {\left[\|\varphi(0)\|_{E}^{2}+2 \sum_{i \in Z} G\left(u_{i 0}\right)\right] e^{-\sigma t} } \\
& +\frac{1}{\alpha \sigma}\|g\|^{2}\left(1-e^{-\sigma t}\right) .
\end{aligned}
$$

But

$$
\begin{aligned}
\sum_{i \in Z} G\left(u_{i 0}\right) & =\sum_{i \in Z} \sum_{j=0}^{m} \frac{a_{j}}{2 j+2} u_{i 0}^{2 j+2} \\
& \leq f^{\prime}(\|u(0)\|) \cdot\|u(0)\|^{2}
\end{aligned}
$$

then,

$$
\begin{aligned}
\|\varphi\|_{E}^{2} \leq & {\left[\|\varphi(0)\|_{E}^{2}+2 f^{\prime}(\|u(0)\|) \mid \cdot\|u(0)\|^{2}\right] e^{-\sigma t} } \\
& +\frac{1}{\alpha \sigma}\|g\|^{2}\left(1-e^{-\sigma t}\right)
\end{aligned}
$$

From (20), for any initial data $\varphi(0)=\left(u_{0}, v_{0}\right)^{T} \in E$, then the solution $\varphi(t)=(u(t), v(t))^{T}$ is bounded for all $t \in[0,+\infty)$, that is, the solution $\varphi(t)$ exists globally on $[0,+\infty)$, maps $\left\{S(t)_{t \geq 0}\right\}$ defined by (11) form a semigroup on $E$. Inequality (19) implies that the semigroup $\{S(t)\}_{t \geq 0}$ possesses a bounded absorbing set in $E$.

LEMMA 3 If $g \in \ell^{2}$, then there exists a bounded ball $O=O_{E}\left(0, r_{0}\right)$, centered at $O$ with radius $r_{0}$, such that for every bounded set $B$ of $E$, there exists
$T(B) \geq 0$ such that

$$
S(t) B \subset O, \quad \forall t \geq T(B)
$$

where $r_{0}^{2}=(2 / \alpha \sigma)\|g\|^{2}$.

Therefore, there exists a constant $T_{0} \geq 0$ depending on $O$ such that

$$
S(t) O \subset O, \quad \forall t \geq T_{0}
$$

\section{GLOBAL ATTRACTOR}

Let $H$ be a complete metric space and $\{S(t), t \geq 0\}$ be a continuous semigroup on $H$.

Definition 1 A set $X$ of $H$ is called a global attractor for the semigroup $\{S(t), t \geq 0\}$ if (i) $X$ is invariant set, i.e., $S(t) X=X, \forall t \geq 0$. (ii) $X$ is a compact set. (iii) $X$ attracts any bounded set of $H$, i.e., for any bounded set $B \subset H, d(S(t) B, X)=$ $\sup _{x \in S(t) B} \inf _{y \in X} d(x, y) \rightarrow 0$ as $t \rightarrow \infty$.

To obtain the existence of a global attractor for the semigroup $\left\{S(t)_{t \geq 0}\right\}$ associated with (8) on $E$. We need prove the asymptotic compactness of $\left\{S(t)_{t \geq 0}\right\}$.

LEMMA 4 If $g \in \ell^{2}$ and $\varphi(0)=\left(u_{0}, v_{0}\right) \in O$, then $\forall \eta>0$, there exists $T(\eta)$ and $K(\eta)$ such that the solution $\varphi(t)=\left(\varphi_{i}\right)_{i \in Z}=\left(\left(u_{i}(t)\right),\left(v_{i}(t)\right)\right)_{i \in Z} \in E$ of problem $(8), v(t)=\dot{u}(t)+\varepsilon u(t)$, satisfies

$$
\begin{aligned}
\sum_{|i| \geq K(\eta)}\left\|\varphi_{i}(t)\right\|_{E}^{2}= & \sum_{|i| \geq k(\eta)}\left[\left|(B u(t))_{i}\right|^{2}+\lambda\left|u_{i}(t)\right|^{2}\right. \\
& \left.+\left|v_{i}(t)\right|^{2}\right] \leq \eta, \quad \forall t \geq T(\eta)
\end{aligned}
$$

where $(B u(t))_{i}=u_{i+1}(t)-u_{i}(t)$

Proof Choosing a smooth function $\theta \in C^{1}\left(R^{+}, R\right)$ satisfies:

$$
\begin{cases}\theta(s)=0, & 0 \leq s \leq 1 \\ 0 \leq \theta(s) \leq 1, & 1 \leq s \leq 2 \\ \theta(s)=1, & s \geq 2\end{cases}
$$


then there exists a constant $C_{0}$ such that $\left|\theta^{\prime}(s)\right| \leq C_{0}$ for $s \in R^{+}$.

Let $\varphi(t)=(u(t), v(t))=\left(\varphi_{i}\right)_{i \in Z}=\left(\left(u_{i}(t)\right), \quad\left(v_{i}\right.\right.$ $(t)))_{i \in Z}$ be a solution of (8), where $v(t)=$ $\dot{u}(t)+\varepsilon u(t), \varphi_{i}=\left(u_{i}, v_{i}\right), \varepsilon$ is as in (7).

Let $k$ be a fixed integer and set $w_{i}=\theta$ $(|i| / k) u_{i}, z_{i}=\theta(|i| / k) v_{i}, \quad y=(w, z)=\left(\left(w_{i}\right),\left(z_{i}\right)\right)_{i \in Z}$. Taking the inner product $(\cdot, \cdot)_{E}$ of (8) with $y$, we have

$$
(\dot{\varphi}, y)_{E}+(C \varphi, y)_{E}=(F(\varphi), y)_{E} .
$$

It is possible to check that

$$
(\dot{\varphi}, y)_{E}=\frac{1}{2} \frac{d}{d t} \sum_{i \in Z} \theta\left(\frac{|i|}{k}\right)\left\|\varphi_{i}\right\|_{E}^{2}
$$

where

$$
\begin{aligned}
\left\|\varphi_{i}\right\|_{E}^{2} & =\left|(B u)_{i}\right|^{2}+\lambda\left|u_{i}\right|^{2}+\left|v_{i}\right|^{2} \\
& =\left|u_{i+1}-u_{i}\right|^{2}+\lambda\left|u_{i}\right|^{2}+\left|v_{i}\right|^{2},
\end{aligned}
$$

and

$$
\begin{aligned}
(C \varphi, y)_{E}= & \varepsilon(B u, B w)-(B v, B w)+\lambda \varepsilon(u, w) \\
& -\lambda(v, w)+(A u, z)+\lambda(u, z) \\
& +\varepsilon^{2}(u, z)-\varepsilon(v, z)+(h(v-\varepsilon u), z),
\end{aligned}
$$

$$
\begin{array}{r}
(B u, B w)(t)=\sum_{i \in Z}\left\{\left[\theta\left(\frac{|i+1|}{k}\right)-\theta\left(\frac{|i|}{k}\right)\right]\right. \\
\left(u_{i+1}-u_{i}\right) u_{i+1}+\theta\left(\frac{|i|}{k}\right) \\
\left.\left(u_{i+1}-u_{i}\right)^{2}\right\} \\
\geq-\frac{4 C_{0} r_{0}^{2}}{k}+\sum_{i \in Z} \theta\left(\frac{|i|}{k}\right)\left(u_{i+1}-u_{i}\right)^{2}, \\
\forall t \geq T_{0}, \\
(B v, B w)=\sum_{i \in Z}\left[\theta\left(\frac{|i+1|}{k}\right)\right. \\
\left(v_{i+1}-v_{i}\right) u_{i+1} \\
\left.-\theta\left(\frac{|i|}{k}\right)\left(v_{i+1}-v_{i}\right) u_{i}\right],
\end{array}
$$

$$
\begin{gathered}
(B u, B z)=\sum_{i \in Z}\left[\theta\left(\frac{|i+1|}{k}\right)\left(u_{i+1}-u_{i}\right) v_{i+1}\right. \\
\left.-\theta\left(\frac{|i|}{k}\right)\left(u_{i+1}-u_{i}\right) v_{i}\right], \\
(B u, B z)-(B v, B w)=\sum_{i \in z}\left(\theta\left(\frac{|i+1|}{k}\right)-\theta\left(\frac{|i|}{k}\right)\right) \\
\left(u_{i+1} v_{i}-u_{i} v_{i+1}\right) \\
\geq-\sum_{i \in Z} \frac{\left|\theta^{\prime}\left(\tau_{i}\right)\right|}{k} \\
\geq-\frac{4 C_{0} r_{0}^{2}}{k}, \quad \forall t \geq T_{0} . \\
(u, w)=\sum_{i \in Z} \theta\left(\frac{|i|}{k}\right) u_{i}^{2}, \\
(v, w)=\sum_{i \in Z} \theta\left(\frac{|i|}{k}\right) u_{i} v_{i}=(u, z), \\
(v, z)=\sum_{i \in Z} \theta\left(\frac{|i|}{k}\right) v_{i}^{2}, \\
\varepsilon^{2}(u, z)+(h(v-\varepsilon u), z) \\
\geq \alpha \sum_{i \in Z} \theta\left(\frac{|i|}{k}\right) u_{i}^{2}-\varepsilon(\alpha-\varepsilon) \\
\sum_{i \in Z} \theta\left(\frac{|i|}{k}\right) u_{i} v_{i},
\end{gathered}
$$

thus,

$$
\begin{aligned}
& (C \varphi, y) \geq-\frac{8 C_{0} r_{0}^{2}}{k}+\sum_{i \in Z} \theta\left(\frac{|i|}{k}\right)\left[\sigma\left\|\varphi_{i}\right\|_{E}^{2}+\frac{\alpha}{2}\left|v_{i}\right|^{2}\right], \\
& \forall t \geq T_{0} . \\
& (F(\varphi), y)_{E}=-(f(u), z)+(g, z) \\
& (f(u), z) \geq \frac{d}{d t} \sum_{i \in Z} \theta\left(\frac{|i|}{k}\right) G\left(u_{i}\right) \\
& +\varepsilon \sum_{i \in Z} \theta\left(\frac{|i|}{k}\right) G\left(u_{i}\right) \\
& (g, z) \leq \frac{\alpha}{2} \sum_{i \in Z} \theta\left(\frac{|i|}{k}\right) v_{i}^{2}+\frac{1}{2 \alpha} \sum_{|i| \geq k} g_{i}^{2} .
\end{aligned}
$$


Putting inequalities (26), (29)-(31) into (25), we obtain

$$
\begin{aligned}
& \frac{d}{d t} \sum_{i \in Z} \theta\left(\frac{|i|}{k}\right)\left[\left\|\varphi_{i}\right\|_{E}^{2}+2 G\left(u_{i}\right)\right] \\
& \quad+\sigma \sum_{i \in Z} \theta\left(\frac{|i|}{k}\right)\left[\left\|\varphi_{i}\right\|_{E}^{2}+2 G\left(u_{i}\right)\right] \\
& \leq \frac{8 C_{0} r_{0}^{2}}{k}+\frac{1}{\alpha} \sum_{|i| \geq k} g_{i}^{2} .
\end{aligned}
$$

Since $g \in \ell^{2}$, then $\forall \eta>0$, there exists $K(\eta)$ such that

$$
\frac{8 C_{0} r_{0}^{2}}{k}+\frac{1}{\alpha} \sum_{|i| \geq k} g_{i}^{2} \leq \eta, \quad \forall k \geq K(\eta)
$$

by Gronwall's inequality,

$$
\begin{gathered}
\sum_{i \in Z} \theta\left(\frac{|i|}{k}\right)\left[\left\|\varphi_{i}\right\|_{E}^{2}+2 G\left(u_{i}\right)\right] \leq e^{-\sigma\left(t-T_{0}\right)} \\
\sum_{i \in Z} \theta\left(\frac{|i|}{k}\right)\left[\left\|\varphi_{i}\left(T_{0}\right)\right\|_{E}^{2}+2 G\left(u_{i}\left(T_{0}\right)\right)\right]+\frac{\eta}{\sigma} \\
\leq e^{-\sigma\left(t-T_{0}\right)} r_{0}^{2}\left(1+2 M_{0}\right)+\frac{\eta}{\sigma}, \quad \forall t \geq T_{0} .
\end{gathered}
$$

where $M_{0}=\left|f^{\prime}\left(r_{0}\right)\right|$. Taking

$$
T(\eta)=\max \left\{T_{0}, T_{0}+\frac{1}{\sigma} \ln \frac{\sigma}{\eta}\left(1+2 M_{0}\right) r_{0}^{2}\right\}
$$

then for $t \geq T(\eta)$ and $k \geq K(\eta)$, we have

$$
\sum_{|i| \geq 2 k}\left\|\varphi_{i}\right\|_{E}^{2} \leq \sum_{i \in Z} \theta\left(\frac{|i|}{k}\right)\left\|\varphi_{i}\right\|_{E}^{2} \leq \frac{2 \eta}{\sigma}
$$

which implies Lemma 4. The proof is completed.

LeMma 5 If $g \in \ell^{2}$, then the semigroup $\{S(t)\}_{t \geq 0}$ is asymptotially compact in $E$, that is, if $\left\{\varphi_{n}\right\}$ is bounded in $E$ and $t_{n} \rightarrow+\infty$, then $\left\{S\left(t_{n}\right) \varphi_{n}\right\}$ is precompact in $E$.

Proof Let $\left\{\varphi_{n}\right\} \subset E=\ell_{\lambda}^{2} \times \ell^{2}$ be bounded, assume that $\left\|\varphi_{n}\right\|_{E} \leq r$ for some positive constant $r, n=1,2, \ldots$ By Lemma 3 , there exists $T_{r}$ such that

$$
S(t) \varphi_{n} \subset O, \quad \forall t \geq T_{r}
$$

where $O$ is the absorbing set. By $t_{n} \rightarrow+\infty$, there exists $N_{1}(r)$ such that $t_{n} \geq T_{r}$ if $n \geq N_{1}(r)$, thus,

$$
S\left(t_{n}\right) \varphi_{n} \subset O, \quad \forall n \geq N_{1}(r)
$$

Since $E$ is a Hilbert space and by (34), there exists $\varphi_{0} \in E$ and a subsequence of $\left\{S\left(t_{n}\right) \varphi_{n}\right\}$ (denoted still by $\left.\left\{S\left(t_{n}\right) \varphi_{n}\right\}\right)$ such that

$$
S\left(t_{n}\right) \varphi_{n} \rightarrow \varphi_{0} \quad \text { weakly in } E .
$$

In what follows, the convergence here is a strong one, i.e., $\forall \eta>0$, there exists $N(\eta)$ such that

$$
\left\|S\left(t_{n}\right) \varphi_{n}-\varphi_{0}\right\|_{E} \leq \eta, \quad \forall n \geq N(\eta) .
$$

For $\eta>0$, by Lemma 4 and (33), there exist $K_{1}(\eta)$, $T(\eta)$ such that

$$
\left.\sum_{|i| \geq K_{1}(\eta)} \|\left(S(t)\left(S\left(T_{r}\right)\right) \varphi_{n}\right)\right)_{i} \|_{E}^{2} \leq \frac{\eta^{2}}{8}, \quad t \geq T(\eta),
$$

By $t_{n} \rightarrow+\infty$, there exists $N_{2}(r, \eta)$ such that $t_{n} \geq T_{r}+T(\eta)$ if $n \geq N_{2}(r, \eta)$, hence,

$$
\begin{aligned}
& \sum_{|i| \geq K_{1}(\eta)}\left\|\left(S\left(t_{n}\right) \varphi_{n}\right)_{i}\right\|_{E}^{2} \\
& \quad=\sum_{|i| \geq K_{1}(\eta)}\left\|\left(S\left(t_{n}-T_{r}\right) S\left(T_{r}\right) \varphi_{n}\right)_{i}\right\|_{E}^{2} \leq \frac{\eta^{2}}{8} .
\end{aligned}
$$

Again, since $\varphi_{0} \in E$, there exists $K_{2}(\eta)$ such that

$$
\sum_{|i| \geq K_{2}(\eta)}\left\|\left(\varphi_{0}\right)_{i}\right\|_{E}^{2} \geq \frac{\eta^{2}}{8}
$$

Let $K(\eta)=\max \left\{K_{1}(\eta), K_{2}(\eta)\right\}$, by (35),

$$
\left(\left(S\left(t_{n}\right) \varphi_{n}\right)_{i}\right)_{|i| \leq K(\eta)} \rightarrow\left(\left(\varphi_{0}\right)_{i}\right)_{|i| \leq K(\eta)}
$$

$$
\text { strongly in } R_{\lambda}^{2 K(\eta)+1} \times R^{2 K(\eta)+1}, n \rightarrow+\infty \text {, }
$$


that is, there exists $N_{3}(\eta)$ such that

$$
\sum_{|i| \leq K(\eta)}\left\|\left(S\left(t_{n}\right) \varphi_{n}\right)_{i}-\left(\varphi_{0}\right)_{i}\right\|_{E}^{2} \leq \frac{\eta^{2}}{2} \quad \forall n \geq N_{3}(\eta) .
$$

Setting $\quad N(\eta)=\max \left\{N_{1}(r), N_{2}(r, \eta), N_{3}(\eta)\right\}, \quad$ from (36) - (38), then for $n \geq N(\eta)$

$$
\begin{aligned}
\left\|S\left(t_{n}\right) \varphi_{n}-\varphi_{0}\right\|_{E}^{2} & =\sum_{|i| \leq K(\eta)}\left\|\left(S\left(t_{n}\right) \varphi_{n}\right)_{i}-\left(\varphi_{0}\right)_{i}\right\|_{E}^{2} \\
& +\sum_{|i|>K(\eta)}\left\|\left(S\left(t_{n}\right) \varphi_{n}\right)_{i}-\left(\varphi_{0}\right)_{i}\right\|_{E}^{2} \\
\leq & \left.\frac{\eta^{2}}{2}+2 \sum_{|i|>K(\eta)}\left(\| S\left(t_{n}\right) \varphi_{n}\right)_{i}\left\|_{E}^{2}-\right\|\left(\varphi_{0}\right)_{i} \|_{E}^{2}\right) \\
\leq & \eta^{2} .
\end{aligned}
$$

The proof is completed.

As a direct consequence of Lemmas 3, 5 and Theorem I. 1.1 of [8], we obtain the existence of a global attractor for semigroup $\{S(t)\}_{t \geq 0}$.

Theorem 1 If $g \in \ell^{2}$, then the semigroup $\{S(t)\}_{t \geq 0}$ associated with (8) possesses a global attractor $\beta$ in $E$.

Remark Since the solutions of problem (8) are backward unique in time, the invariance of the global attractor $B$ means

$$
S(t) B=ß \quad \text { for } t \in R .
$$

We can consider the mapping $S_{0}(t):\left(u_{0}, u_{10}\right)^{T} \rightarrow$ $(u(t), \dot{u}(t))^{T} \in \ell^{2} \times \ell^{2}$ associated with problem (3) in the space $\ell^{2} \times \ell^{2}$ with the usual inner product and norm. Since $S_{0}(t)=R_{-\varepsilon} S(t) R_{\varepsilon}, R_{\varepsilon}=\left(\begin{array}{ll}1 & 0 \\ \varepsilon & 1\end{array}\right)$ is an isomorphism on $\ell^{2} \times \ell^{2}$ and $\{S(t)\}_{t \geq 0}$ possesses a global attractor $B$ in $E$, the global attractor of $\left\{S_{0}(t)\right\}_{t \geq 0}$ in $E$ is $R_{-\varepsilon} B$, which implies that $\left\{S_{0}(t)\right\}_{t \geq 0}$ possesses a global attractor in $\ell^{2} \times \ell^{2}$ because $\ell^{2} \times \ell^{2}$ and $E$ have the same elements and their norms are equivalent.

\section{References}

[1] Chate, H. and Courbage, M. (Eds.) (1997). Lattice Systems, Proceedings of the workshop on Lattice Dynamics, Paris, 21 - 23 June, 1995, Physic D, 103(1-4), 1-612.

[2] Chow, S. N., Mallet-Parat, J. and Shen, W. (1998). Thaveling waves in lattice dynamical systems, J. Diff. Equa., 149(2), 248-291.

[3] Jiang, M. (1999). The entropy formula for SRB-measures of lattice dynamical systems, J. Statist. Phys., 95(3-4), $791-803$.

[4] Shen, W. (1996). Lifted lattices, hyperbolic structures, and topological disorders in coupled map lattices, SIAM J. Appl. Math., 56(5), 1379-1399.

[5] Yu, J. (1998). Collective behavior of coupled map lattices with asymmetrical coupling, Phys. Lett. A, 240(1-2), $60-64$.

[6] Shengfan Zhou (1999). On dimension of the global attractor for damped nonlinear wave equation, J. Math. Phys., 40, 1432-1438.

[7] Hale, J. K., "Asymptotic behavior of dissipative systems", Amer. Math. Soc., providence, Rhode Island 1988.

[8] Temam, R., "Infinite-dimensional dynamical systems in mechanics and physics", Appl. Math. Sci., 68, SpringerVerlag, New York, 1988.

[9] Feireisl, E. (1995). Global attractors for semilinear damped wave equations with supercritical exponent, J. Diff. Equa., 116, 431-447.

[10] Ghidaglia, J. M. and Temam, R. (1987). Attractors for damped nonlinear wave equations, J. Math. Pure Appl., 66, 273-319.

[11] Karachalios, N. I. and Starrakakis, N. M. (1999). Existence of a global attractor for semilinear dissipative wave equations on $R^{n}, J$. Diff. Equa., 157, 183-205.

[12] Feireisl, E. (1997). Long time behavior and convergence for semilinear wave equations on $R^{n}, J$. Dynam. Diff. Equa., 9(1), 133-155. 


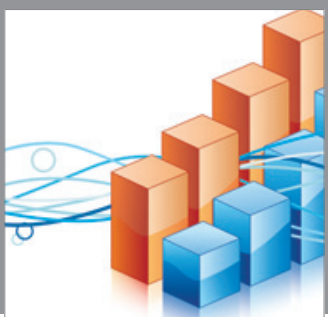

Advances in

Operations Research

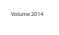

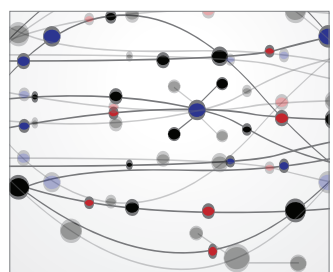

\section{The Scientific} World Journal
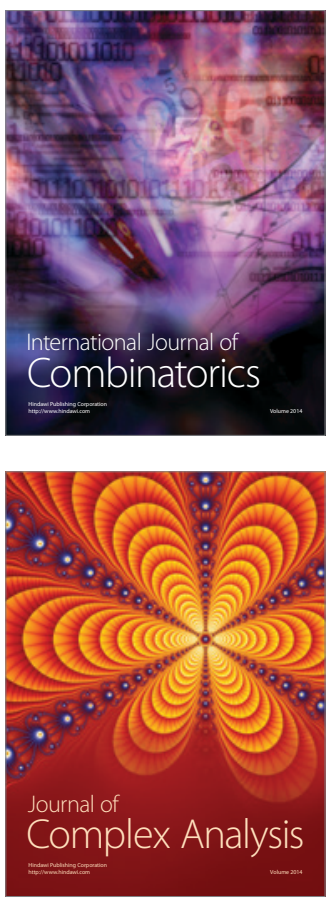

International Journal of

Mathematics and

Mathematical

Sciences
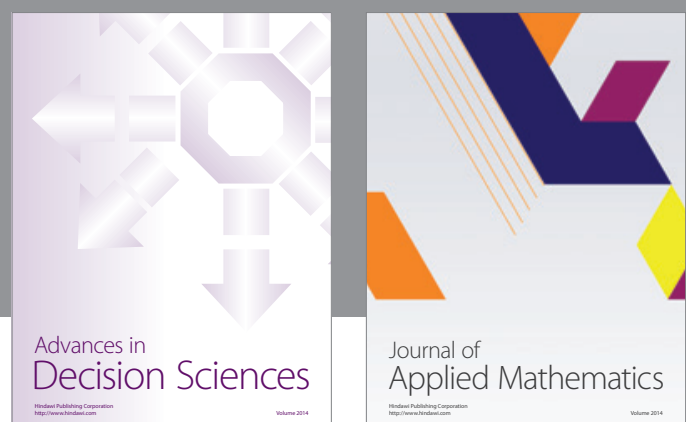

Journal of

Applied Mathematics
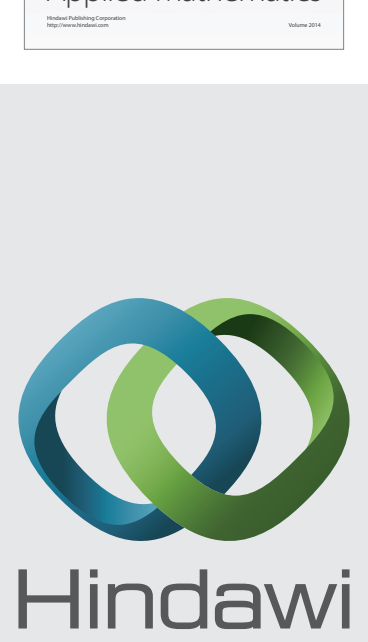

Submit your manuscripts at http://www.hindawi.com
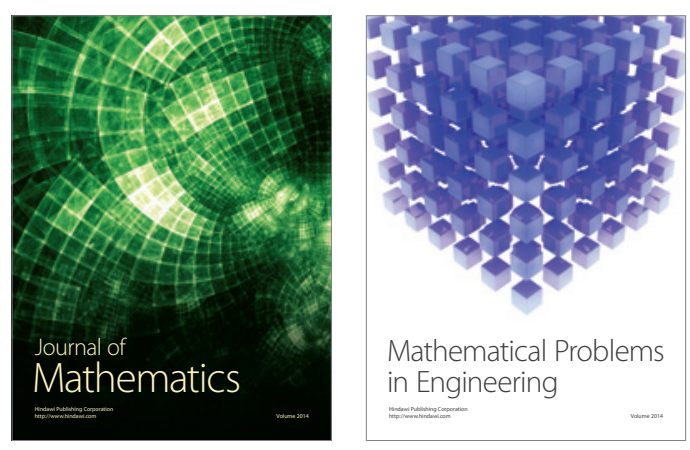

Mathematical Problems in Engineering
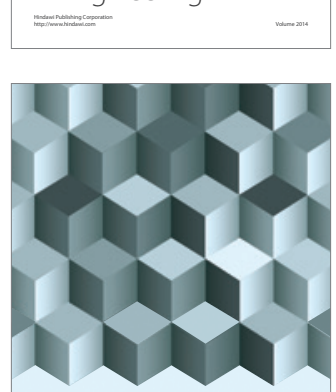

Journal of

Function Spaces
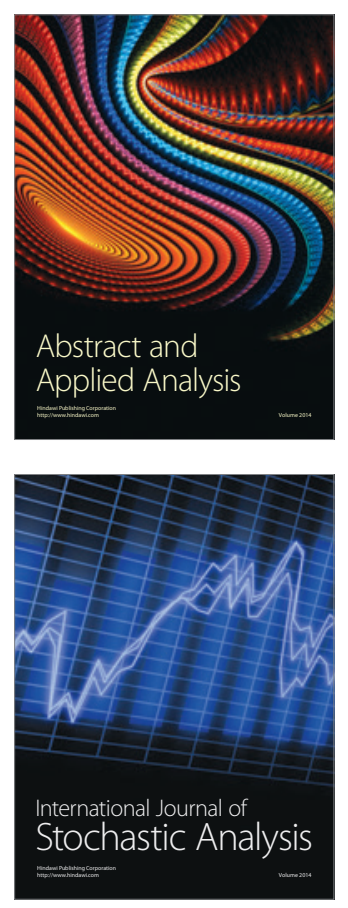

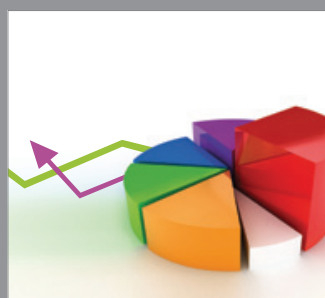

ournal of

Probability and Statistics

Promensencen
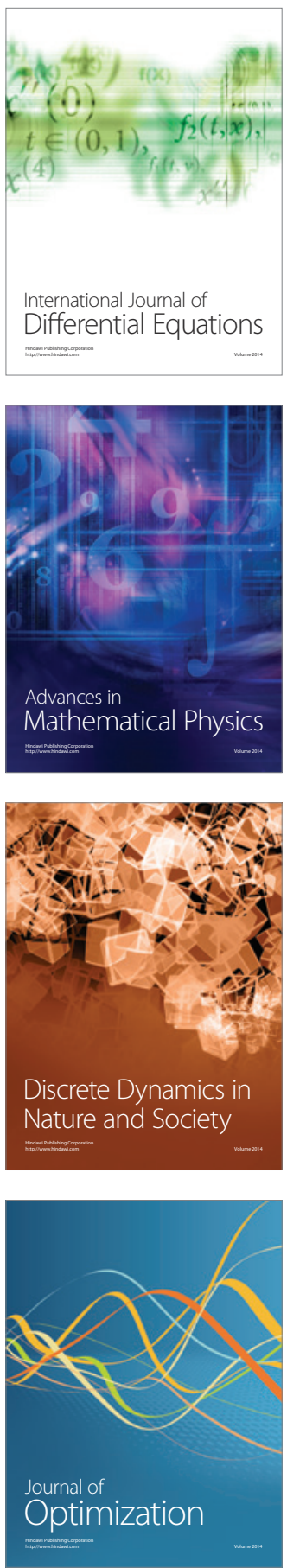